\title{
Erfolg bei metastasierendem Melanom
}

Speziell behandelte dendritische Zellen können offenbar erfolgreich Melanome zerstören. In einem ersten klinischen Versuch zeigte sich bei drei von sieben Patienten ein Erfolg. Ein Krebskranker mit ehemals metastasierendem Melanom befindet sich seit vier Jahren in Remission. Zwei weitere Patienten sprachen auf die Therapie an - bei ihnen dauerte es knapp ein Jahr bis zur Tumorprogression.

Dem Team um Beatriz Carreno von der Universität in St. Louis, MO/USA, ist es gelungen, mit einer Art zellulären

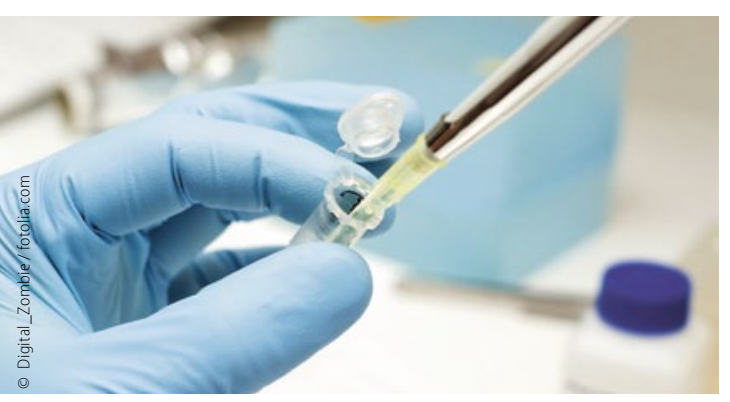

Dendritische Zellen werden mit GP40-

Ligand und Gamma-Interferon behandelt.
Impfung das körpereigene Immunsystem gezielt auf Melanomzellen anzusetzen. Sie entnahmen den Patienten dazu zunächst dendritische Zellen. Diese Antigen-präsentierenden Zellen sind in der Lage, T-Zellen in einer Weise zu aktivieren, dass diese eine Armee von zytotoxischen Effektorzellen aufstellen, mit denen sich wirksam Tumoren bekämpfen lassen.

Damit dendritische Zellen die Entwicklung der T-Zellen zu zytotoxischen Tc1Zellen steuern, benötigen sie mehrere Signale: neben dem Antigen auch Interleukin 12p70 (IL-12p70). Dies produzieren sie unter bestimmten Bedingungen selbst, nämlich dann, wenn ihr GP40-Rezeptor belegt und gleichzeitig Gamma-Interferon vorhanden ist.

In ihrem Versuch haben die Forscher um Carreno nun unreife dendritische Zellen ex vivo mit einem GP40-Liganden und Gamma-Interferon behandelt, um die Produktion des gewünschten Interleukins anzuregen. Dann ließen sie die Zellen differenzieren und traktierten sie mit Melanom-Antigenen. Derart aktivierte Zellen infundierten sie den Patienten - jeweils sechsmal im Abstand von drei Wochen.

Drei der Patienten bildeten tatsächlich massiv Tc1-Killerzellen gegen den Tumor. Dabei hing sowohl die T-Zell-Antwort als auch der klinische Erfolg von der produzierten Menge an IL-12p70 ab: Je mehr die Zellen produzierten, umso länger dauerte es bis zur Tumorprogression.

Bei drei der vier Non-Responder bildeten sich allerdings Tc2- statt der erwünschten Tc1-Zellen. Diese Kommunikationsprobleme ließen sich durch Zugabe weiterer Faktoren beheben, sodass auch bei diesen Patienten die tumorspezifische Tc1-Zellen-Produktion angeregt werden konnte. Über den klinischen Erfolg dieser Korrektur machten die Forscher um Carreno jedoch noch keine Angaben.

Das Interleukin direkt zu verabreichen hätte zu starke unerwünschte Effekte. Der Weg über dendritische Zellen könnte also eine neue Strategie zur Entwicklung von wirksamen Tumorvakzinen sein.

Thomas Müller

Carreno BM et al. IL-12p70-producing patient DC vaccine elicits Tc1-polarized immunity. J Clin Invest. 2013;123(8):3383-94.

\section{Immuntests sind Enzymtest klar überlegen}

In einer deutschen Studie mit mehr als 2.000 Patienten wurden mit immunologischen Tests doppelt so viele Darmkrebserkrankungen und fast dreimal so viele Krebsvorstufen entdeckt wie mit dem enzymatischen Test. Hermann Brenner und Sha Tao vom Deutschen Krebsforschungszentrum (DKFZ) in Heidelberg verglichen die Aussagekraft dreier immunologischer fäkaler Okkultbluttests zum Nachweis von Hämoglobin mithilfe von Antikörpern (iFOBT) mit der des enzymatischen Hämoccult-Tests (gFOBT). Beim Hämoccult-Test lagen die positiven Vorhersagewerte für Darmkrebs, Adenome und frühe Vorstufen bei 4,5\%, 17,1\% und 30,6\%. Bei den immunologischen Tests dagegen waren die Werte für Darmkrebs und Adenome etwa doppelt bzw. dreimal so hoch.

Die Sensitivität für Darmkrebserkrankungen insgesamt lag beim enzymati- schen Test bei 33,3\%, die für Adenome bei $8,6 \%$, und die für frühe Vorstufen bei $5,5 \%$. Bei allen drei Veränderungen betrug die Spezifität des Tests mehr als $95 \%$ : Bei 95 von 100 Probanden mit negativer Koloskopie fiel auch der Hämoccult-Test negativ aus. Wesentlich besser waren die immunologischen Ergebnisse. Mit ihnen konnten zwischen 53\% und $74 \%$ der Krebserkrankungen sowie zwischen $20 \%$ und knapp $26 \%$ der Adenome aufgespürt werden. Zudem lag die Spezifität mit bis zu $97 \%$ noch etwas höher als beim enzymatischen Test. PeterLeiner

Brenner $\mathrm{H}$ et al. Superior diagnostic performance of faecal immunochemical tests for haemoglobin in a head-to-head comparison with guaiac based faecal occult blood test among 2235 participants of screening colonoscopy. Eur J Cancer. 2013 May 22. [Epub ahead of print]. kurz notiert

Forschungsstandort Deutschland

DNA-Spuren zeigen Krebsursache +++ Wissenschaftler des Deutschen Krebsforschungszentrums (DKFZ) arbeiteten an einem internationalen Projekt zu Erforschung spezifischer Muster krebserregender DNA-Mutationen mit [Alexandrov LB et al. Nature. 2013 Aug 14. (Epub ahead of print)]. Die Forscher analysierten fast fünf Millionen Mutationen bei über 7.000 Krebsarten und fanden mehr als 20 spezifische Mutationsmuster. Die Ergebnisse sind wichtig zur Entwicklung effektiverer Präventions- und Therapieformen.

Kombinationstherapie per Simulation +++ An der Charité in Berlin gelang es, die Wirksamkeit unterschiedlicher Kombinationstherapien bei Darmkrebs durch Computersimulation prognostizierbar zu machen [Klinger B et al. Mol Syst Biol. 2013;9:673]. Die entwickelten Computermodelle simulieren zelluläre Netzwerke verschiedener Darmkrebszellen. Die Simulationsannalyse ermöglicht die Ermittlung bestimmter Moleküle, die fehlerhafte Netzwerksignale senden, und eine zur gezielten Ausschaltung geeignete Medikamentenkombination. 